\title{
RETRACTED ARTICLE: Multi-layered multi-exemplar affinity propagation for temporal clustering of human motion
}

\author{
Shao-Mei $\mathrm{Li}^{1} \cdot$ Hui $\mathrm{Yu}^{2} \cdot$ Ya-Wen Wang ${ }^{1} \cdot \mathrm{Chao} \mathrm{Gao}^{1}$
}

Received: 4 January 2015 / Revised: 16 March 2015 / Accepted: 2 April 2015 /

Published online: 18 April 2015

(C) Springer Science+Business Media New York 2015

The publisher, in agreement with the Editor-in-Chief, has retracted this article because it contains large portions of text that have been duplicated from a previously published article: Wang Z, Wang S, Ji Q (2013) Capturing complex spatio-temporal relations among facial muscles for facial expression recognition. Proc. IEEE Conference Computer Vision Pattern Recognition 3422-3429.

The online version of this article contains the full text of the retracted article as electronic supplementary material.

The online version of this article (doi:10.1007/s11042-015-2608-3) contains supplementary material, which is available to authorized users.

Shao-Mei Li

lishaomei_may@126.com

Hui $\mathrm{Yu}$

hui.yu@port.ac.uk

Ya-Wen Wang

wyw@mail.ndsc.com.cn

Chao Gao

gc@mail.ndsc.com.cn

1 National Digital Switching System Engineering and Technological Research and Development Center, Zhengzhou, China

2 University of Portsmouth, Portsmouth, UK 